\title{
Modeling Dynamic Ductility: An Equation of State for Porous Metals
}

Jeffrey Colvin

July 30, 2007

American Physical Society Topical Conference on Shock Compression of Condensed Matter (APS/SCCM) Kohala Coast, $\mathrm{HI}$, United States June 25, 2007 through June 29, 2007 
This document was prepared as an account of work sponsored by an agency of the United States Government. Neither the United States Government nor the University of California nor any of their employees, makes any warranty, express or implied, or assumes any legal liability or responsibility for the accuracy, completeness, or usefulness of any information, apparatus, product, or process disclosed, or represents that its use would not infringe privately owned rights. Reference herein to any specific commercial product, process, or service by trade name, trademark, manufacturer, or otherwise, does not necessarily constitute or imply its endorsement, recommendation, or favoring by the United States Government or the University of California. The views and opinions of authors expressed herein do not necessarily state or reflect those of the United States Government or the University of California, and shall not be used for advertising or product endorsement purposes. 


\title{
MODELING DYNAMIC DUCTILITY: AN EQUATION OF STATE FOR POROUS METALS
}

\author{
Jeffrey D. Colvin ${ }^{1}$ \\ ${ }^{1}$ Lawrence Livermore National Laboratory, L-356, P.O. Box 808, Livermore CA 94551
}

\begin{abstract}
Enhanced heating from shock compression of a porous material can potentially suppress or delay cracking of the material on subsequent expansion. In this paper we quantify the expected enhanced heating in an experiment in which a sector of a thin cylindrical shell is driven from the inside surface by SEMTEX high explosive ( $\sim 1 \mu \mathrm{s}$ FWHM pressure pulse with peak pressure $\sim 21.5 \mathrm{GPa})$. We first derive an analytical equation of state (EOS) for porous metals, then discuss the coupling of this EOS with material elastic-plastic response in a 2D hydrocode, and then discuss the modeling of the HE experiment with both fully dense and $10 \%$ porous Ta and a Bi/Ta composite. Finally, we compare our modeling with some recent experimental data.
\end{abstract}

Keywords: shocks in solids, equation of state, porous materials, deformation and ductility

PACS: 62.20.Fe, 62.50. $+\mathrm{p}, 64.10 .+\mathrm{h}, 81.05 . \mathrm{Rm}$

\section{INTRODUCTION}

Enhanced heating from shock compression of a porous material can potentially suppress or delay cracking of the material on subsequent expansion. The objective of this work is to quantify the expected enhanced heating from shock compression of a porous material. We do this by deriving an analytical equation of state (EOS) for porous metals and then coupling it to material elastic-plastic response in a hydrodynamics code. This approach obviates the need for expensive and time-consuming direct numerical simulation (DNS) of pore collapse. DNS is impractical for material design, but can be used to determine the effects of pore collapse on material constitutive properties, as can atomistic simulations [1].

\section{EQUATION OF STATE DERIVATION}

We start with a Gruneisen EOS, and modify it by a pressure multiplier as a function of porosity, as given by Zel'dovich and Raizer [2]. We integrate the pressure over the total volume change to obtain the specific energy as a function of compression. The resulting pressure and specific energy are the sum of three components: lattice compression, lattice thermal, and electron thermal, as follows.

Lattice compression -

$$
\begin{aligned}
& P_{c}=\rho_{0} c_{0} \mu\left[1+\left(1-\gamma_{0} / 2\right) \mu-b \mu^{2} / 2\right] / \\
& \left\{[1-(S-1) \mu]^{2}(K-k-k \mu)\right\} \\
& E_{c}=P_{c}[k(1+\mu)-1] /\left[2 \rho_{0}(1+\mu)\right] .
\end{aligned}
$$

Lattice thermal -

$$
\begin{aligned}
& P_{t}=\left(\gamma_{0}+b \mu\right)(1+\mu) \rho_{0} c_{p} T \\
& E_{t}=3 N_{A}[(Z+1) / A] T .
\end{aligned}
$$

Electron thermal - 


$$
\begin{aligned}
& P_{e}=(1 / 4) \rho_{0} \beta_{0} T^{2} \\
& E_{e}=\beta_{0} T^{2} /[2(\mu+1)] .
\end{aligned}
$$

Here, we have used a form of the Gruneisen EOS for which the ratio of the thermal pressure to the thermal energy is a constant - neglecting nonlinear terms in the Hugoniot, where (values in parentheses are the values for Ta): $\mu=\rho / \rho_{0}-1=$ fractional compression; $\rho_{0}=$ initial solid density $\left(16.69 \mathrm{~g} \mathrm{~cm}^{-3}\right) ; \rho_{00}=$ initial porous density; $\mathrm{k}=$ $\rho_{0} / \rho_{00}=$ porosity coefficient; $c_{0}=$ bulk sound speed $(3.41 \mathrm{~km} / \mathrm{s}) ; \gamma_{0}=$ initial Gruneisen gamma (1.67); b $=$ coefficient of volume dependence of gamma (0.42); $\mathrm{S}=$ slope of Hugoniot (1.2); $\mathrm{K} \sim 2$ for metals; $\mathrm{c}_{\mathrm{p}}=$ material specific heat $(0.135 \mathrm{~J} / \mathrm{g}-\mathrm{K})$; $\mathrm{N}_{\mathrm{A}}=$ Avogadro's number; $\mathrm{Z}=$ material average ionization (0); $\mathrm{A}=$ material atomic weight (180.95 $\left.\mathrm{g} \mathrm{mole}^{-1}\right) ; \beta_{0}=$ electronic heat capacity (370 ergs $/ \mathrm{g}-\mathrm{K}^{2}$ ).

For modest temperatures and compressions, the electron thermal component is small compared to the lattice component, less than $2 \%$, and can be neglected (although we include it in our simulations).

It is clear from the first of equations 1 that it takes more pressure to compress a material to a particular compression $(\mu>1)$ than to compress the full-density material to the same compression. This is because of the compressional energy gained in collapsing the pores. Thus, the lattice compressional energy $E_{c}$ is the sum of two components: energy from collapsing the pores and energy from compressing the lattice. The energy gained in collapsing the pores is just the difference between the total $\mathrm{PdV}$ compressional energy $\mathrm{E}_{\mathrm{c}}$ (integrated along the Rayleigh line), which is the second of equations 1 above, and the lattice compressional energy $\mathrm{E}_{\mathrm{cp}}$ (integrated along the Hugoniot):

$$
E_{c p}=\int_{\mu_{00}}^{\mu}\left[P_{c} /\left\{\rho_{0}(1+\mu)^{2}\right\}\right] d \mu .
$$

We substituted the first of equations 1 above into equation 4 and performed the integration analytically by rewriting the integrand as a sum of partial fractions, each of which could be separately integrated analytically.

Note that this model is applicable only for modest compressions and porosities, and we assume that the pores close completely. Since we apply the model only in regimes where the pressure is well above the material strength, the assumption of complete pore closure is probably a good one, although there may be some dependence on pore size [1]. Thus, we do not model the low-pressure phase of pore collapse, so our model is different than the various so-called P- $\alpha$ (pressure-distension) [3] and $\varepsilon-\alpha$ (strain-distension) [4] EOS models for porous materials. Our basic approach is somewhat similar to that of $\mathrm{Wu}$ and Jing $[5,6]$, but we basically scale the solid-density EOS along an isochoric path rather than an isobaric path as they do. Our derivation is similar to that of Grady [7], but the new feature in our work is that we do the integration of equation 4 to get the component of the total lattice energy resulting from the pore collapse.

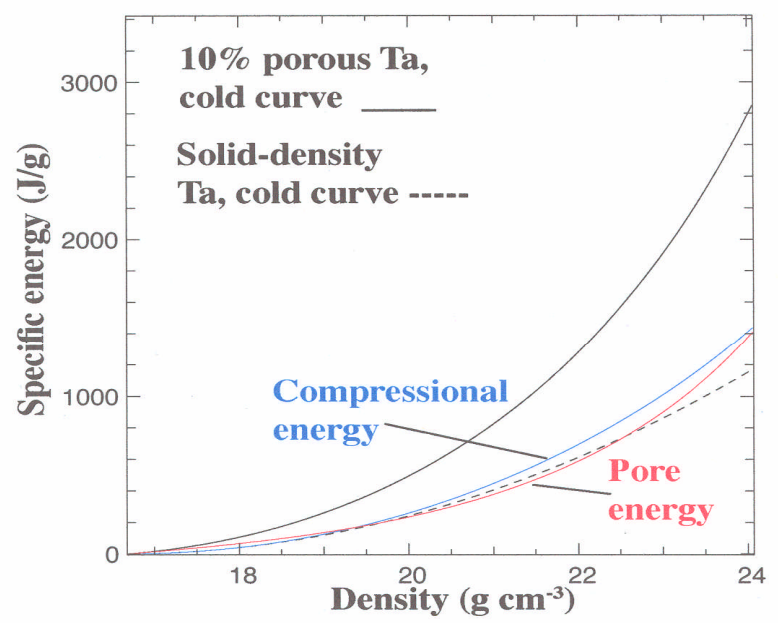

Figure 1. Specific energy as a function of density for $10 \%$ porous $\mathrm{Ta}$ and for full-density $\mathrm{Ta}$, from evaluating equations 1 and 4 .

Inserting the values for $\mathrm{Ta}$ for the various parameters in the EOS equations, we find that a 300 -kbar shock compresses $10 \%$ porous Ta to only slightly lower density than it does solid-density Ta. The added compressional heating of $10 \%$ porous $\mathrm{Ta}$, however, approximately doubles the specific 
energy of the material at $\sim 10 \%$ compression, as shown in Fig. 1. Will there be the same heating, though, when accounting for the material's elasticplastic response? This question was addressed by implementing this analytical EOS model in a hydrodynamics code with material strength, and comparing simulations with experimental data, as discussed in the next section.

\section{SIMULATION OF THE EXPERIMENT AND COMPARISON TO DATA}

In the as-designed experiment, SEMTEX high explosive (HE) is used to drive a pressure pulse into a sector of a $1.5-\mathrm{mm}$-thick cylindrical metal shell from the inside surface. The 70.6-mm-square Ta sheet was shaped into a cylindrical sector of radius $75 \mathrm{~mm}$ and chord length $68 \mathrm{~mm}$. The thicker $\mathrm{HE}$ was formed into a cylindrical sector so that its outer surface was in contact with the inner surface of the metal. The HE was lit at one end of the cylindrical axis. The pressure history at the $\mathrm{HE} /$ metal interface was computed separately by a 3D arbitrary Lagrange-Eulerian code. The pressure rises in $\sim 0.3 \mu$ s to a peak of $\sim 21.5 \mathrm{GPa}$, has a FWHM of $\sim 1 \mu \mathrm{s}$, and is followed by a long tail-off to zero pressure at $\sim 5 \mu \mathrm{s}$.

This pressure drive was used in separate calculations using the $2 \mathrm{D}$ radiation-hydrodynamics code Lasnex [8] to compare the elastic-plastic response of a Ta shell with and without porosity. In both simulations we used the Steinberg-Guinan constitutive model [9] with initial yield strength $0.77 \mathrm{GPa}$ and with work hardening. We do not account for the local change in constitutive properties that results from the pore collapse.

Accounting for the elastic-plastic response of the material, we find that the peak pressure in the initially porous $\mathrm{Ta}$ is only slightly higher than in the initially full-density Ta. The pressure pulse transits the shell in $\sim 0.75 \mu \mathrm{s}$, after which the wave reverberates as the shell expands and decompresses. At the time of release the entire initially porous shell is at $\sim 900 \mathrm{~K}$, compared to the initially full-density shell at $\sim 400 \mathrm{~K}$. The wave rings out in about three shell transit times, after which the shell temperature is approximately constant as the shell expands outward and goes into tension. Fig. 2 shows the simulated mass-averaged temperature history of the shell for the two cases.

As seen in Fig. 2, the temperature of the initially porous Ta during the long expansion

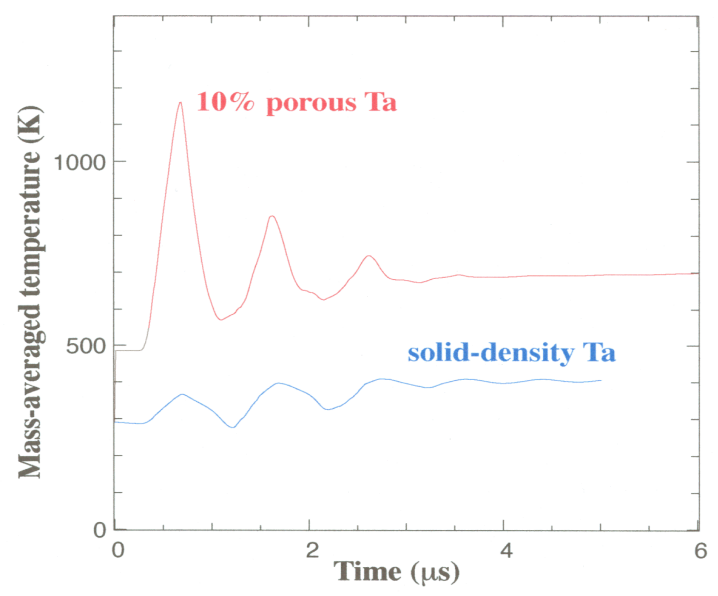

Figure 2. Simulated mass-averaged temperature history of initially $10 \%$ porous Ta shell (upper curve) and initially full-density Ta shell (lower curve) driven by SEMTEX HE.

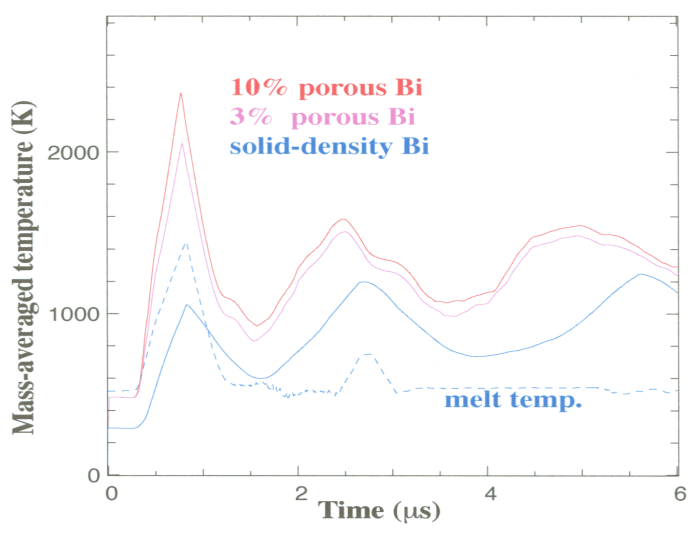

Figure 3. Simulated mass-averaged temperature history of (solid curves, top to bottom) $10 \%$ porous, $3 \%$ porous, and full-density $\mathrm{Bi}$ shell driven by SEMTEX HE, compared to Bi melt temperature (dashed curve).

phase, $\sim 700 \mathrm{~K}$ (compared to $\sim 400 \mathrm{~K}$ for the initially full-density shell), is still much lower than the Ta melt temperature, $\sim 4340 \mathrm{~K}$. We do not know if this enhanced heating is enough to change the ductility of the material by a measurable amount. Accordingly, to get closer to or above melt 
for our first experimental test of this concept of dynamic ductility enhancement we chose to use $\mathrm{Bi}$ (actually, a Bi-Ta composite [10]) since it has a much lower melt temperature.
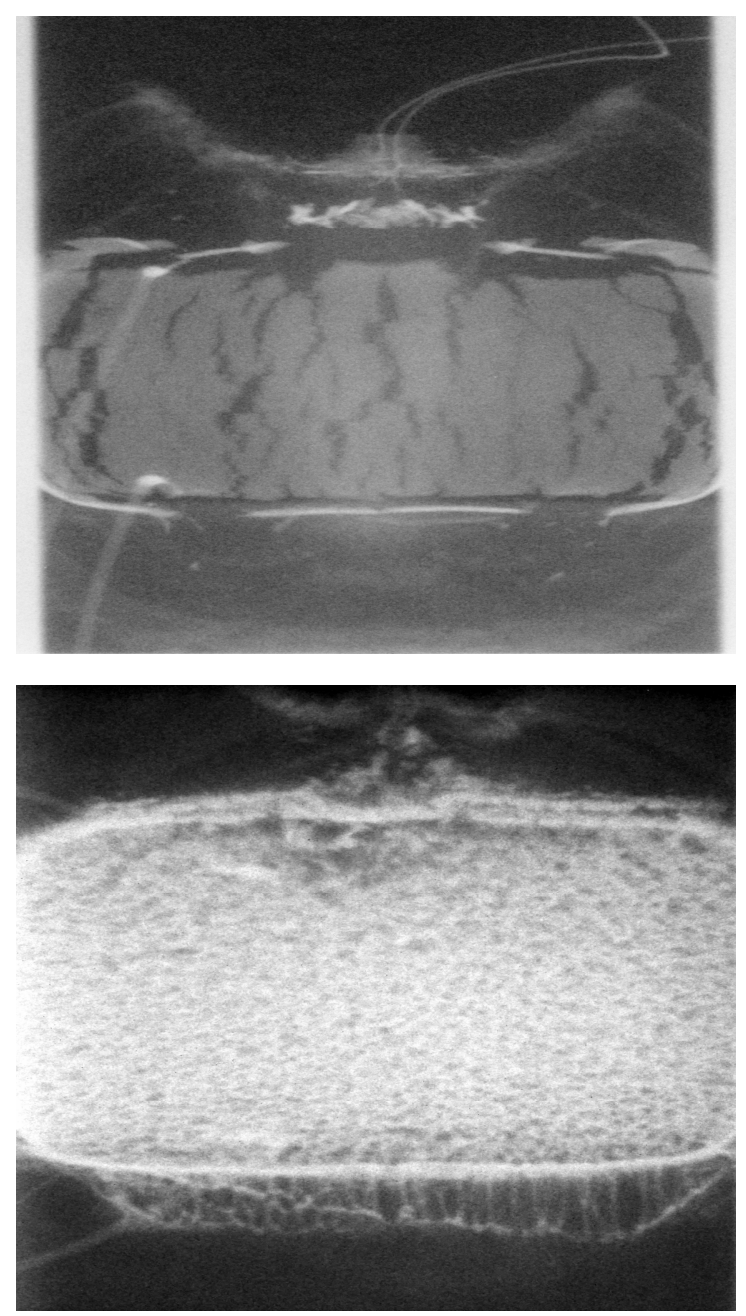

Figure 4. Radiograph of (top) full-density stainless steel shell at $35 \mu \mathrm{s}$, strain 0.57; (bottom) $3 \%$ porous $\mathrm{Bi} / \mathrm{Ta}$ shell at $41 \mu \mathrm{s}$, strain 0.57

As seen in Fig. 3, which shows the simulated massaveraged temperature history of the Bi shell for an initial $10 \%$ porosity, an initial $3 \%$ porosity, and initially full density compared to the $\mathrm{Bi}$ melt temperature, we see that an initially porous Bi shell melts on compression under these $\mathrm{HE}$ drive conditions, unlike an initially full-density Bi shell, which melts only on release. Note that since the wave speed in the $\mathrm{Bi}$ is slower than in the Ta, the wave takes longer to ring out, and there is less of a difference between the temperatures in the expansion phase after about $6 \mu \mathrm{s}$.

The experimental radiographs (Fig. 4) --looking face-on to the outside surface of the outwardly expanding shell --- show that fulldensity metal cracks on HE-driven expansion while the porous Bi shell does not. The two radiographs were taken on different shots at different times, but at the same strain. The mottling shown in the $\mathrm{Bi}$ image is actually much lower contrast than the contrast in the stainless steel image. A Fast Fourier Transform analysis on the images shows no dominant spatial scales in the $\mathrm{Bi}$ mottling. We suggest that this structure may be a signature of inhomogeneous melt.

This work was performed under the auspices of the US Department of Energy by UC LLNL under Contract W-7405-Eng-48.

Support received from LDRD

Project \#06-SI-005. The author acknowledges the contributions of Geoff Campbell, James Stolken, Rich Becker, Eduardo Bringa, Paul DeMange, and Peter Martin of LLNL to this work.

\section{REFERENCES}

1. Erhart, P., Bringa, E. M., Kumar, M., and Albe, K., Phys. Rev. B, vol. 72, 052104, 2005.

2. Zel'dovich, Ya. B. and Raizer, Yu. P., Physics of Shock Waves and High-Temperature Hydrodynamic Phenomena, (Academic Press, New York, 1966), pp. 712-716.

3. Carroll, M. M. and Holt, A. C., J. Appl. Phys., vol. 43, pp. 1626-1636, 1972.

4. Wünneman, K., Collins, G. S., and Melosh, H. J., Icarus, vol. 180, pp. 514-527, 2006.

5. Wu, Q., and Jing, F., Appl. Phys. Lett., vol. 67, pp. 49-51, 1995.

6. Wu, Q., and Jing, F., J. Appl. Phys., vol. 80, pp. 4343-4349, 1996.

7. Grady, D., unpublished report (Applied Research Associates, Inc., Albuquerque, NM 87110, 2005).

8. Zimmerman, G. B. and Kruer, W. L., Comments Plasma Phys. Control. Fusion, vol. 2, pp. 51-61, 1975.

9. Steinberg, D. J, Cochran, S. G., and Guinan, M. W., J. Appl. Phys., vol. 51, pp. 1498-1504, 1980.

10. Martin, L. P., Hodge, A. M., and Campbell, G. H., Scripta Mat., vol. 57, pp. 229-232, 2007. 